\title{
RDA: el alcance internacional del nuevo Código de Catalogación
}

\section{RDA: The international reach of the new Code of Cataloguing}

Paola Andrea Picco GÓMEZ

\section{RESUMEN}

Este artículo resume los grandes cambios conceptuales que sustentan al nuevo Código de Catalogación, cuanto a herramienta que va a facilitar la normalización y permitir alcanzar el tan anhelado control bibliográfico universal. El artículo comienza con una revisión histórica de la catalogación y los inicios de la cooperación, para luego describir los modelos conceptuales que sustentarán al nuevo Código de Catalogación y enfatizar las ventajas que ofrecerá al usuario. Se explica como se organizará este nuevo Código y los cambios en la terminología utilizada, para finalizar analizando el alcance internacional que han tenido las Reglas y que tendrá el nuevo Código. Corresponde destacar que, si bien las Reglas de Catalogación Angloamericanas han sido traducidas a varios idiomas y adoptadas por varios países como Código de Catalogación, solo los cuatro países anglosajones, que integran el Joint Steering Committee (Estados Unidos, Canadá, Reino Unido y Australia) han tenido un papel protagónico en la elaboración y revisión de las mismas.

Palabras claves: catalogación; reglas de catalogación; descripción bibliográfica; control de autoridades.

\section{A B STRACT}

The most important changes which support the new Cataloguing Code are presented as a tool which facilitates the standardization and as a way to reach the so-wished Universal Bibliographic Control. A historic review of Cataloguing, including the beginning of cooperation in the subject, is included. The conceptual models which support the new Code and the benefits which it will bring to the users are analysed. The new organization of the Code and the new terminology adopted are explained; finishing with the analysis of the international reach of AACR and the new Code. The article stresses the fact that although AACR has been translated into several languages and adopted by many countries as their Cataloguing Code, the elaboration and revision of the Code has been conducted only by the four countries members of the Joint Steering Committee for Revision of AACR (USA, Canada, United Kingdom and Australia).

Keywords: cataloguing; cataloguing code; bibliographic description; authority control.

\footnotetext{
1 Magíster en Arte - Universidad de Manitoba Canadá. Licenciada en Bibliotecología - Escuela Universitaria de Bibliotecología y Ciencias Afines - Universidad de la Repúblic. Docente Procesos Técnicos I - Escuela Universitaria de Bibliotecología y Ciencias Afines - Universidad de la República. Dirección: Emilio Frugoni, 1429, CP 11200, Montevideo, Uruguay. E-mail: <paopicco@adinet.com.uy>.

Recebido em 22/5/2007 e aceito para publicação em 31/8/2007.
} 


\section{NTRODUCCIÓN}

La corrida hacia la elaboración de un código de catalogación internacional comenzó hace varios años. Se puede establecer como inicio la Conferencia de Paris (1961), en la cual expertos en catalogación se pusieron de acuerdo en una serie de principios, que permitieron establecer vínculos de cooperación en la elaboración de reglas de catalogación de aplicación internacional.

Otro hito importante fue la reunión organizada por la IFLA en Copenhague, en 1969, que tuvo como resultado la elaboración de la International Standard Bibliographic Description (ISBD), que, como afirma Manning, "es un elemento clave en el esfuerzo de conseguir el Control Bibliográfico Universal" (Manning, 1998, p.2).

En 1967 se publicó la primera edición de las Reglas de Catalogación Angloamericanas y once años después, la segunda edición que fue revisada en 1988. Si bien se han publicado enmiendas para la segunda edición, las RCAA2 no han logrado estar al nivel de los cambios tecnológicos y de las repercusiones que han tenido sobre la producción editorial y el intercambio de información. En 1997 se organizó una conferencia internacional sobre los principios y futuros desenvolvimientos de las Reglas de Catalogación Angloamericanas, en la cual, entre las conclusiones finales, surgió la recomendación de realizar un análisis lógico de los principios y estructuras en las que se basan estas reglas.

Como resultado de este análisis se esperaba que, finalmente, se definiera el esquema teórico que sustentaría las AACR3 (en español RCAA3). En el año 2004 se publicó el primer borrador y se designó como editor a Tom Delsey, un canadiense con mucha experiencia en catalogación. La designación del editor marcó un cambio importante en la estructura de las reglas porque se abandonó la idea original de publicar las AACR3, y se propuso un nuevo esquema que fue denominado RDA (Resource Description and Access). El borrador de las AACR3 fue, además, duramente criticado por su pobreza en la cobertura de los documentos digitales.

El título propuesto es ilustrativo de los cambios conceptuales que propone descripción y acceso de recursos. En primer lugar hay un cambio en el objeto de catalogación, pasa del ítem de las RCAA2 al recurso; el segundo gran cambio es que incluye en la segunda parte del Código, directrices e instrucciones para el control de los puntos de acceso. Las RDA definen al "recurso" como "cualquier objeto identificable de información, sea de naturaleza tangible o intangible" (Resource..., 2006, p.1). El nuevo Código retoma al ítem, pero como una de las entidades del universo bibliográfico (modelo FRBR) a describir, y este concepto se aplica específicamente a la copia o copias que existen de determinada manifestación en la colección. Con la incorporación de una definición más amplia del objeto de catalogación, se hace un esfuerzo por superar la orientación de la descripción de los documentos impresos que existía en las RCAA2.

\section{El código tendrá dos partes principales además} del Glosario, apéndices e índice. La primera parte va a estar dedicada a la descripción de los recursos, cuyo modelo conceptual va a ser el esquema de los Requisitos Funcionales para los Registros Bibliográficos (FRBR). La segunda parte, Acceso, apuesta en un cambio significativo con respecto a las prácticas corrientes de catalogación e introduce reglas para el control de los puntos de acceso, conocido hasta ahora como control de autoridades. La inclusión de reglas para el control de puntos de acceso es un aporte real sin precedentes al control bibliográfico universal. Como afirma Gorman "There is a sense in which authority control and bibliographic control are coterminous - two sides of the same coin"2 (Gorman, 2004, p. 12).

La parte dedicada al acceso se apoya en el modelo conceptual Requisitos funcionales para los datos de autoridades (FRAD), conocido anteriormente como (FRANAR) Requisitos funcionales para el registro de autoridades.

\section{Los modelos conceptuales que sustentan el nuevo Código}

Los modelos conceptuales que sustentan el nuevo Código de Catalogación se basan en el modelo entidad-relación, que es comúnmente utilizado para el diseño de bases de datos. Las FRBR se originaron con el objetivo de brindar un marco conceptual a la catalogación que contemple los cambios tecnológicos que han impactado al universo bibliográfico. Es además, una propuesta de simplificación de la catalogación descriptiva y de desenvolver herramientas

\footnotetext{
${ }^{2}$ Hay un sentido en el cual el control de autoridad y el control de autoridad y el control bibliográfico son términos complementarios - los dos lados de una misma moneda.
} 
que permitan catalogar todo tipo de documentos. El modelo define tres grupos de entidades, atributos para cada una de ellas y las relaciones que se pueden establecer entre las mismas. La aplicación de este modelo permite reflejar las relaciones que existen en el universo bibliográfico. Al respecto Tillet dice "[...] el universo bibliográfico consiste en diversas entidades que se relacionan entre sí y pueden ser descritas mediante elementos de información (o atributos)" (Tillet, 2003, p.6).

El modelo identifica las entidades que son de interés para los usuarios y que se deben incluir en los registros bibliográficos, los atributos que describen a cada entidad y las relaciones que se pueden establecer entre ellas favoreciendo así las tareas que debe realizar el usuario. Estas tareas son: búsqueda, identificación, selección y la obtención de recursos de información.

Las FRBR definen a las entidades como los objetos que son de interés para el usuario en el universo bibliográfico y las divide en tres grupos. El primer grupo incluye a las entidades que representan productos del esfuerzo intelectual o artístico (obra, expresión, manifestación e ítem). El segundo grupo incluye a las entidades que son responsables del contenido intelectual y artístico de las entidades del primer grupo (persona y entidad corporativa). Las entidades del tercer grupo representan el contenido de una de las entidades del primer grupo que es la obra. Es decir cubre los aspectos temáticos de la obra (concepto, objeto, evento y lugar).

Cada una de las entidades definidas en el modelo tiene sus atributos que son los elementos de información que describen a cada entidad, y por los cuales los usuarios formulan sus búsquedas. Ejemplos de algunos atributos son: para la entidad obra: título de la obra, fecha de la obra, etc. , para la entidad expresión: título de la expresión, fecha de la expresión, idioma de la expresión, etc.

Este modelo permite establecer relaciones entre las distintas entidades definidas, una obra por ejemplo, con sus distintas expresiones y manifestaciones. Un caso concreto sería "El Quijote de la Mancha" (la obra) que se relaciona con las distintas traducciones, revisiones, ediciones (expresión). Toda obra y expresión se materializa en una manifestación. Por lo tanto podemos tener a "El Quijote de la Mancha" que es la obra, en español que corresponde a la entidad "expresión", esta obra y expresión se materializa en un libro, en un video o en un DVD, dicha materialización corresponde a la entidad "manifestación". El ítem, la cuarta entidad del primer grupo, es un ejemplar de una determinada manifestación, en este caso un ítem de "El Quijote de la Mancha". Se hace necesario llegar a este nivel de detalle, por que los distintos ejemplares en una colección pueden tener diferencias, un ejemplar mutilado, un ejemplar con una dedicatoria, etc.

Un catálogo que aplique las FRBR va a permitir que el usuario vea en un único registro las distintas expresiones, manifestaciones e ítems de una misma obra. De esta forma se facilita la tarea de búsqueda, identificación, selección y ubicación que debe realizar el usuario. (Este ejemplo es válido para las relaciones entre las entidades del primer grupo).

Las entidades del grupo dos son responsables del contenido intelectual y artístico de las obras, expresiones y manifestaciones. Por lo tanto, se pueden establecer relaciones con el autor de una obra y los traductores de las distintas expresiones y los editores de las distintas manifestaciones de la misma obra. Los autores, editores, traductores estarían reunidos bajo la entidad " persona » si son individuos y bajo la entidad " entidad corporativa "si son grupos de individuos u organizaciones. En el caso de las entidades del grupo tres, cubren los aspectos temáticos de la obra, dichas entidades son plausibles de relacionarse con las expresiones o manifestaciones de determinada obra. A su vez las entidades del tercer grupo se pueden relacionar con las del segundo grupo, cuando una persona o entidad corporativa es un tema. Las entidades del grupo 3 no van a estar cubiertas en el nuevo Código.

El modelo conceptual que sustenta el Control de Autoridades FRAD (Functional requirements for authority data) fue concebido como una extensión de los FRBR. El objetivo del mismo fue la definición de requisitos funcionales a incluirse en los registros de autoridades para facilitar el intercambio de datos de autoridades. Este modelo esta todavía en discusión, no existiendo hasta la fecha nada más que los borradores, que han ido evolucionando, como lo refleja el cambio en el título del mismo. Comenzó llamándose FRANAR (Functional requirements and numbering of authority record), luego FRAR (Functional Requirements for Authority Record) para finalizar en FRAD (Functional Requirement for Authority Data). Este modelo retoma a las entidades ya definidas por las FRBR (persona, entidad corporativa, obra, expresión, manifestación, item, 
concepto, objeto, evento, lugar) y agrega las entidades familia, nombre, identificador, punto de acceso, reglas y agencia.

La entidad familia en este modelo conceptual pasa a hacer parte del segundo grupo definido en las FRBR. Familia se define como las personas relacionadas por el nacimiento, casamiento, adopción o un estado legal similar. Las entidades definidas en el modelo FRBR y la entidad familia del modelo FRAD son todas entidades bibliográficas. A partir de ellas se elaboran los puntos de acceso por los cuales los usuarios recuperan la información, y por lo tanto, deben ser controlados. Las otras cinco entidades que definen este modelo tienen que ver con el proceso de creación de los registros de autoridades. Define al Nombre como la palabra o palabras por la cual se conoce una entidad. Identificador es el número, código, palabra frase u otro símbolo por el cual se asocia únicamente a una entidad y se utiliza para diferenciarlo de las otras entidades con características similares. Punto de acceso, el término bajo el cual un registro bibliográfico o de autoridad es recuperado. Reglas, como las instrucciones a seguir para la formulación de los puntos de acceso. Agencia, como la organización responsable de crear y modificar los puntos de acceso. A partir de los nombres por los cuales las entidades del grupo uno, dos o tres pueden ser conocidas o los identificadores que se le asignan a cada una de estas entidades, se crean los puntos de acceso que deben ser controlados por el Control de los puntos de acceso.

Mientras las FRBR reflejan las relaciones existentes en el universo bibliográfico, este modelo refleja las relaciones que existen entre las autoridades en ese universo bibliográfico, y es así que se pueden establecer relaciones entre una persona con su familia o con una entidad corporativa a la cual pertenece.

\section{El alcance del nuevo Código de catalogación y sus objetivos}

El Código define como objeto de catalogación a los "recursos". El Código incluye directrices e instrucciones que van a permitir al usuario descubrir los distintos recursos dentro de una colección. El concepto de descubrir implica: encontrar, identificar, seleccionar y obtener. Estas cuatro funciones están definidas en el modelo conceptual FRBR y según Tillet son "tareas del usuario, las cosas que consideramos que el usuario desea hacer con respecto al universo bibliográfico" (Tillet, 2003, p.5). Por lo tanto, con la aplicación del nuevo código de catalogación, el catálogo permitirá al usuario encontrar a la entidad o entidades, identificarla, poder seleccionarla y obtenerla. Una entidad puede ser una obra, un autor, una entidad corporativa, una edición de una obra, una traducción, etc.

Con la aplicación del modelo entidad-relación, se van a establecer relaciones y asociaciones entre los distintos registros que van a facilitar la tarea del usuario. Para tomar como ejemplo, con las RCAA2 el catalogador debe optar por realizar un único registro para un título con dos manifestaciones, (dos ítems con dos menciones de ediciones distintas) o crear registros separados. Si se lleva en cuenta el interés del usuario, la primera es la mejor solución. El usuario en un único registro tiene todos los elementos para seleccionar e identificar el documento que busca. Sin embargo, esta solución no contempla el principio de que cada edición debe tener un registro por separado. Con la aplicación de los atributos y relaciones esta situación se resuelve fácilmente. A través de las relaciones que el catalogador establece, el usuario puede ver todas las manifestaciones del mismo título a la vez (en este caso, obra) y este despliegue facilita enormemente la función de identificación y selección que realiza el usuario. Existen ya algunos catálogos donde se aplican las FRBR y las relaciones que se establecen permiten que se recuperen todos los recursos que estén vinculados. Por ejemplo las diferentes ediciones de una misma obra.

A continuación se incluye un ejemplo de catálogo FRBR, donde aparecen dos manifestaciones de una misma obra. Si se observa la información se despliega en orden jerárquico, la obra (Wide Sargasso Sea), la expresión (texto en inglés), la manifestación (los detalles referentes a esa edición que son los atributos). En este caso faltaría la cuarta entidad del primer grupo (obra, expresión, manifestación, ítem), que es la que permite obtener la obra e incluye atributos referentes a su ubicación física y al número de ejemplares.

Hay que destacar que para aplicar este modelo se requiere un trabajo consecuente de parte de los catalogadores, para que el sistema funcione y sea coherente. Cabe preguntarse entonces si los catalogadores están preparados para hacer frente a 
estos cambios, aunque vale recordar que desde el catálogo manual, se vienen estableciendo relaciones para vincular los documentos dentro de una colección. Tenemos por lo menos tres ejemplos para citar: el caso de la fichas de referencia de "véase además", la utilización del título uniforme y el asiento secundario nombre-título. En el caso de la referencia "véase además", la función es advertir que hay documentación asociada a un nombre o título que se está buscando. La regla 26.1C1 establece "Haga una referencia de véase además a partir de un encabezamiento de nombre o de título a otro encabezamiento de nombre, título uniforme o título relacionado" (Reglas..., 2004, 26.1C1, p.26-2).

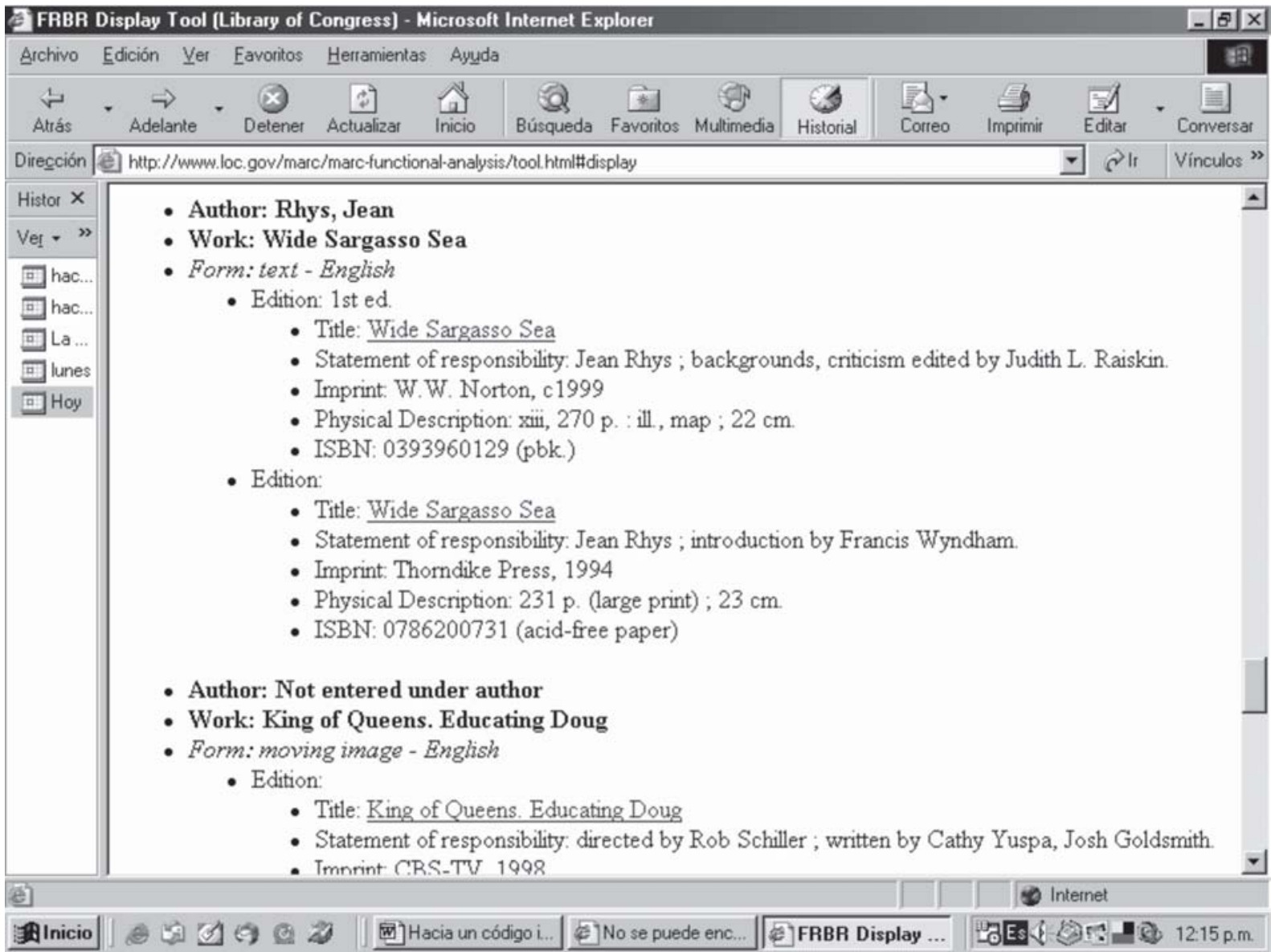

Figura 1. Ejemplo tomado de la Library of Congresss. FRBR Display Tool.

El título uniforme cuyo objetivo es:

agrupar todos los asientos catalográficos para una obra cuando diversas manifestaciones (por. Ej. Ediciones, traducciones) de ésta han aparecido bajo diversos títulos; para identificar una obra cuando el título por el cual se la conoce difiere del título propiamente dicho del ítem que se cataloga [...] (Reglas..., 2004, 25.1.A, p.25-5). 
Haga un asiento secundario bajo el encabezamiento de una obra que tiene estrecha relación con la obra que se cataloga (véanse 21.8 - 21.28 como guía para casos específicos). En tal caso, el encabezamiento es el de la persona o entidad corporativa o el del título bajo el cual la obra con la que se relaciona se asienta o se asentaría. Si el encabezamiento es de una persona o entidad y el título de la obra con la cual se relaciona difiere del de la obra que se cataloga, agregue al encabezamiento el título de la obra con la cual se relaciona para formar un encabezamiento de asiento secundario de nombre-título. [...] (Reglas..., 2004, 21.30G1, p.21-51).

Como se puede observar en los tres casos citados, el concepto de asociar o establecer vínculos entre las obras relacionadas estaba presente en el espíritu de las RCAA2. El modelo entidad-relación con su terminología y la definición de las distintas entidades con sus correspondientes atributos facilita la tarea. Con estos ejemplos se pretende demostrar, que el concepto de asociar no es ajeno a la práctica corriente de la catalogación.

\section{La estructura del nuevo Código y algunos de los grandes cambios}

El código va a estar estructurado en dos grandes partes; la primera dedicada a la descripción y la segunda al control de los puntos de acceso. Dentro de cada una de estas partes se van a encontrar capítulos. En la parte correspondiente a la descripción, diferente de las RCAA2, los capítulos no van a estar organizados por el tipo de material sino por los distintos atributos utilizados para la descripción de cada recurso (título propiamente dicho, variaciones en el título propiamente dicho, mención de responsabilidad, mención de edición, etc.). Esta primera parte va a dividirse en dos, la primera dedicada a la descripción puramente y la segunda a las relaciones entre las distintas entidades.

Cuanto a la Descripción, un cambio significativo es que no se van a establecer distintos niveles de detalle en la catalogación, como sucede actualmente en las RCAA2. El nuevo código va a identificar los atributos necesarios para incluirlos en un registro de catalogación.
El primer capítulo del Código va a definir los tipos de descripción a realizar, que podrán ser: exhaustiva, analítica o multi-nivel.

Un segundo cambio importante es que no se van a utilizar ni las áreas ni la puntuación prescripta por las ISBD. Sin embargo, uno de los apéndices va a contener información sobre las ISBD para aquellos usuarios que deseen seguir utilizándolas. El nuevo Código no va a prescribir una estructura de registro para la presentación de la información, ya sea descriptiva o de acceso. El Código va a determinar una serie de atributos necesarios para la descripción de los distintos recursos que podrán ser almacenados, y presentados en distintos formatos. Es importante señalar aquí que el Código va a ser independiente de cualquier formato de almacenamiento y presentación de datos.

El primer capítulo de la segunda parte del Código va a incluir directrices generales para establecer los puntos de acceso preferidos y las correspondientes referencias, van haber otros seis capítulos donde se definirán los atributos y sus relaciones con las personas, familias, entidades, lugares, obras, expresiones, manifestaciones e ítem.

Además de la nueva organización dentro de esta sección, el gran aporte es que define atributos para cada una de las entidades, necesarias para establecer el control de los puntos de acceso.

En esta segunda parte se van a incluir además las directrices e instrucciones para la elección de los puntos de acceso. Se van a prescribir los criterios para la elección del punto de acceso primario y los siguientes. El punto de acceso primario viene a sustituir al asiento o encabezamiento principal. Si bien se ha discutido sobre la necesidad de determinar un punto de acceso principal o primario que pasa desapercibido para el usuario en un catálogo, no se pudo eliminar ya que es necesario como criterio de ordenación en las bibliografías. La terminología utilizada va a cambiar también. Se va a incorporar toda la terminología del nuevo modelo conceptual: las entidades, relaciones, atributos. También hay un cambio en cuanto al objeto de catalogación, que fue mencionado previamente. El término encabezamiento va a sustituirse por el de punto de acceso, que ya se venía utilizando en el ámbito de la catalogación. El término control de autoridad, va a pasar a llamarse control de los puntos de acceso, encabezamiento autorizado por punto de acceso preferido, título uniforme va a pasar a ser título 
preferido. Se va a abandonar el término entrada principal por el de punto de acceso primario que fue explicado previamente. $Y$ asiento secundario va a pasar a llamarse punto de acceso secundario. Hay que advertir que se está trabajando sobre los borradores en inglés y las traducciones al español, que están siendo realizadas por la autora de este artículo, por lo tanto pueden aparecer otros términos en español en las traducciones autorizadas de estas reglas. Pero lo que se quiere destacar, es que la nueva terminología que va a utilizar el nuevo Código coincide con la ya utilizada en los catálogos en línea, o en el entorno digital.

\section{El alcance internacional del Código de Catalogación}

Las Reglas de Catalogación Angloamericanas han logrado un alcance internacional, han sido traducidas a 25 idiomas (checo, catalán, griego, ruso, árabe, malasio, chino, danés, finlandés, francés, italiano, japonés, norvego, portugués, español, sueco, turco y urdu, alemán, filipino, ucraniano, vietnamita entre otros). Sin embargo el Comité encargado de revisar las reglas y de la elaboración del nuevo Código (Joint Steering Committee for Revisión of Anglo American Cataloguing Rules) está integrado exclusivamente por organizaciones de cuatro países angloparlantes como son: The American Library Association, The Australian Comité on Cataloguing, The British Library, The Canadian Committee on Cataloguing, CILIP - Chartered Institute of Library and Information Professionals (Reino Unido), y the Library of Congress. En el 2004 y 2006 el Comité entregó distinciones a personas que habían realizado contribuciones a la revisión de las reglas. Durante este periodo se entregaron en total 29 distinciones, todas pertenecientes a instituciones de los 4 países que integran el Joint Steering Committee. El prefacio de la revisión de las RCAA2 afirma que:

La aceptación de las AARC2 continúa creciendo en el mundo. [...] Compartir la catalogación entre las bibliotecas se ha convertido en un componente esencial del planeamiento gerencial, particularmente en las bibliotecas nacionales, alentando a la cooperación internacional y a la armonización de las prácticas catalográficas más allá de la fronteras nacionales. (Anglo-American..., 1998).
Al leer esta afirmación, y al analizar la documentación sobre el Joint Steering Committee se encuentra cierta contradicción, porque finalmente las discusiones y los aportes se dan entre Estados Unidos, Canadá, Reino Unido y Australia. Es a partir de esta información que cabe preguntarse cuál es el verdadero alcance internacional de las reglas. ¿̇Por qué muchos de los países que utilizan las Reglas de Catalogación Angloamericanas no están envueltos en esta discusión? Si verdaderamente se cree que las Reglas de Catalogación Angloamericanas son un instrumento para alcanzar el control bibliográfico universal, sería necesario que todos los países que utilizan las Reglas participen de la discusión y de su actualización. Con el nuevo código la situación es similar. El Joint Steering Committee ha tenido un papel protagónico en el desarrollo de los borradores, realizando consultas a las bibliotecas nacionales de Francia, Alemania, Suecia, Noruega, Canadá, Gran Bretaña, Rusia y España. Ninguna biblioteca nacional de América Latina fue incluida en dicha consulta.

Revisando los prefacios de las ediciones de las RCAA anteriores, surge que en 1974 se realizó una reunión tripartita donde participaron delegados de Estados Unidos, Reino Unido y Canadá, cuyo objetivo fue discutir el proyecto de la $2^{a}$ ed de las RCAA. En esa reunión se fijaron 4 objetivos. El objetivo número 4 es particularmente revelador:

\begin{abstract}
Estimular el interés internacional en las RCAA facilitando su uso en otros países fuera de Estados Unidos, Canadá y el Reino Unido. Este objetivo final se intensificó más tarde, como una condición establecida por el Council on Library Resources, al brindar una contribución al desarrollo de un Código internacional de catalogación (Anglo-American..., 1978)
\end{abstract}

Resulta interesante entonces destacar que parecería que el interés de desarrollar un Código internacional estuvo influenciado por razones económicas, y a ésto se debe agregar el hecho de que el Joint Steering Committee es financiado, principalmente, por los royalties generados por la comercialización de las Reglas.

Todavía faltan dos años para que se publique el nuevo Código en inglés, algún tiempo más para que esté disponible en español. Actualmente se están 
discutiendo los capítulos de la segunda parte. Tal vez haya tiempo suficiente para que las comunidades usuarias de las Reglas puedan reclamar su participación activa en este proceso.

\section{CONCLUSIÓN}

Con la aplicación del modelo entidad relación, los catálogos se convertirán en herramientas verdaderamente útiles en el momento de la identificación y la selección de los recursos. La terminología utilizada en la catalogación será más precisa y adecuada al tipo de recursos que se va a catalogar y al entorno digital. El nuevo modelo conceptual de la catalogación permitirá además, tener un óptimo aprovechamiento de las tecnologías.

A pesar de que muchos países han adoptado las Reglas de Catalogación Angloamericanas como código de catalogación, el proceso de revisión y de actualización está monopolizado por el Joint Steering Committee y los cuatro países anglosajones que lo integran. Parece necesario y urgente redefinir la estructura de funcionamiento de este comité, para integrarlo con otros países con experiencia en elaboración de Códigos y en catalogación, de manera que se pueda tener una visión más amplia y diversa. De esta manera se podría formar un grupo pluralista haciendo posible que el Código sea publicado en varias lenguas simultáneamente y no tener que depender de las traducciones que se hacen del inglés. Quedan dos años todavía para que se publique el nuevo Código, recién se está terminando la discusión sobre el modelo conceptual que va a sustentar la segunda parte (Acceso) y falta por preparar los capítulos dedicados a los puntos de acceso. Es muy importante que las bibliotecas nacionales de América Latina se involucren en el proceso de revisión de estos capítulos, si se pretende lograr un verdadero Código de alcance universal.

\section{REFERÊ NCIAS}

ANGLO-AMERICAN Cataloguing Rules. Preface to the 1998 revision. 1998. Disponible en: <http://www.collectionscanada. ca/jsc/docs/aacr2preface 1998.pdf> Acceso en: 12 mar. 2007.

ANGLO-AMERICAN Cataloguing Rules. Preface to the Anglo American Cataloguing Rules, Second Edition, 1978. 1998. Disponible en: <http://www.collectionscanada.ca/jsc/docs/ aacr2preface 1978. pdf>. Acceso en: 12 mar. 2007.

GORMAN, M. Authority control in the context of bibliographic control in the electronic environment. Cataloging and Classification Quarterly, v.38, n.3-4, p.11-21, 2004.

MANNING, R.W. Las Anglo-American Cataloguing Rules y su futuro. En: 64th IFLA GENERAL CONFERENCE, 64., 1998, Amsterdam. Disponibe en: <http://www.ifla.org/IV/ifla64/083126s.htm >. Acceso en: 15 dic. 2006.

REGLAS de Catalogación Angloamericanas : preparadas bajo la dirección del Joint Steering Committee for Revision of AACR, un comité de la American Library Association ... [et al.] ; tr. y revisión general por Margarita Amaya de Heredia. 2.ed., revisión de 2002, actualización 2003. Bogotá : Rojas Eberhard, 2004.

RESOURCE Description and Access. Scope and Structure, 2006. Disponible en: <http://www.collectionscanada.ca/jsc/docs/5rdascope.pdf>. Acceso en: 2 feb. 2007.

TILLET, B. El modelo FRBR (requerimientos funcionales para registros bibliográficos) una presentación de Dra. Barbara B. Tillet. San José, 2003. Disponible en: <http://www.loc.gov/catdir/cpso/ frbrspan. pdf>. Acceso en: 4 oct. 2005. 